\title{
RESIDENCIA DE PERINATOLOGÍA EN EL HOSPITAL UNIVERSITARIO DE CARACAS (VENEZUELA)
}

\section{Perinatology residence in the Caracas Teaching Hospital, Venezuela}

Libardo Augusto Gómez-Castro, M.D.*, Juan Pérez, M.D.**, Carlos Bermúdez, M.D.**, Cristian Sosa, M.D.*, Freddy Guevara, M.D.***

Recibido: octubre 18/06 - Revisado: febrero 12/07 - Aceptado: febrero 26/07

\section{RESUMEN}

La unidad de perinatología del Hospital Universitario de Caracas, fue fundada en 1975 por los doctores Freddy Guevara y Miguel Yáber; su objetivo ha sido mejorar la salud materno-fetal para la solución de los principales problemas perinatales.

Dentro de sus objetivos específicos, está la formación de subespecialistas en perinatología; la residencia inicia en enero de 1989, bajo la dirección del doctor Guevara.

Actualmente cuenta con una moderna planta física en el Hospital Universitario de Caracas, con tecnología de punta, convenios interinstitucionales y un equipo de profesionales altamente capacitados, continuando con la formación de perinatólogos en Venezuela y Latinoamérica, ingresando para la subespecialización cinco ginecoobstetras por año, durante un período de dos años.

\footnotetext{
* Especialista en ginecología y obstetricia, residente II año perinatología. Hospital Universitario de Caracas, Venezuela, piso 10, Unidad de Perinatología, Caracas (Venezuela). Unidad de medicina materno fetal Fetosur Colombia, Calle 71 \# 41 - 54, Barranquilla, Colombia. Correo electrónico: libardogomezcastro@hotmail.com.

** Especialista Ginecología y Obstetricia, subespecialista Perinatología, docente Unidad Perinatología Hospital Universitario de Caracas.

*** Especialista Ginecología y Obstetricia, subespecialista Perinatología, director Unidad de Perinatología Hospital Universitario de Caracas.
}

El perinatólogo de la unidad está capacitado para prestar servicios de salud en aspectos preventivos, médicos, quirúrgicos y de rehabilitación en sus áreas de especialización; participar en trabajos de investigación en humanos, animales y docencia universitaria.

Palabras clave: residencia perinatología, Hospital Universitario de Caracas, medicina materno fetal.

\section{SUMMARY}

The Caracas Teaching Hospital's perinatology unit was founded in 1975 by doctors Freddy Guevara and Miguel Yaber. Its objective has been to improve foetal maternal health for resolving the main perinatal problems. Training sub-specialists in perinatology lies within its specific objectives.

Dr. Guevara began directing residency in January 1989. The unit currently has a modern plant in the Caracas Teaching Hospital, having cutting-edge technology, inter-institutional agreements and a highly trained team of professionals.

It continues training sub-specialists in perinatal medicine in Venezuela and Latin-America, 5 gynaeco-obstetricians entering the unit for subspecialisation per year (for a 2-year period). 
Perinatologists from the unit are trained to provide health services in preventative, medical, surgical rehabilitation aspects in their specialisation areas, participate in human and animal research work and university teaching.

Key words: perinatology residence, University Hospital of Caracas, maternal foetal medicine.

\section{INTRODUCCIÓN}

Existe actualmente en la práctica de la obstetricia, gran incertidumbre por quién está en capacidad de evaluar y manejar embarazos de alto riesgo, lo cual ha promovido los cursos de acreditación (tamización ecográfica de malformaciones, procedimientos invasivos, evaluación de riesgo del embarazo, ecocardiografía, etc.), pero ¿existen unos lineamientos mínimos para un obstetra?, ¿hasta dónde puede llegar el obstetra?, ¿qué sería competencia exclusiva del perinatólogo?, ¿por quiénes son realizados los cursos de acreditación?

Muy probablemente no tenemos las respuestas a estos interrogantes, pero sí existe hoy día la posibilidad de una educación médica continua, de ahí que nuestro objetivo sea mostrar como está estructurada y qué nivel de entrenamiento se adquiere en la realización de una subespecialidad de perinatología durante dos años con dedicación exclusiva.

Aunque no existe una fecha exacta de cuándo inicia la perinatología como subespecialidad en el mundo, se considera que esta aparece después de la Segunda Guerra Mundial, el primer antecedente se conoce en Francia en donde se crea un plan de incentivos a la reproducción humana, bajo criterios de calidad, en el que la atención integral de la mujer embarazada, como su entorno socio económico y cultural fueron prioridad.

Como subespecialidad se considera que inicia tres décadas atrás, ante la necesidad que existía de disminuir los índices de morbimortalidad materna e infantil, es así como desde entonces se considera una rama de la obstetricia y la neonatología. ${ }^{1}$

Etimológicamente, perinatología significa tratado sobre el conocimiento de todas las situaciones presentes alrededor de un nacimiento; conceptualmente se asume como una rama de la ciencia médica, estrechamente vinculada con la obstetricia y con la neonatología, en virtud de que ambas están comprometidas con el futuro del recién nacido y de su madre. La Organización Panamericana de la Salud la define como la especialidad que se ocupa del cuidado de la mujer embarazada a partir de las 28 semanas de gestación hasta los siete primeros días del recién nacido, solo incluye la morbimortalidad fetal tardía y la neonatal precoz. ${ }^{2}$

Teniendo un concepto más global de la perinatología o actualmente de la medicina materno fetal, podríamos definirla como aquella subespecialidad de la obstetricia que tiene como base garantizar la atención adecuada y oportuna de la mujer embarazada y su producto, en especial embarazos de alto riesgo, que amenacen en grado variable, la integridad del binomio madre-feto, período que se inicia aun con la mujer no embarazada y que culmina con la etapa neonatal precoz. ${ }^{3}$

En Ibero América tenemos la creación como subespecialidad en México en el año de 1983, como el Instituto Nacional de Perinatología dependiente de la Universidad Autónoma de México, ${ }^{4}$ en Uruguay en el año de 1970 se crea el Centro Latinoamericano de Perinatología, dependiente de la Organización Panamericana de la Salud y de la Organización Mundial de la Salud, ${ }^{2}$ en España la Unidad de Perinatología dependiente de la Universidad de Valencia y la Unidad de Diagnóstico Prenatal en el Instituto Dexeus, dependiente de la Universidad de Barcelona, ${ }^{5}$ en Colombia se aprueba la subespecialidad en el año 2002 en convenio de la Clínica Colsanitas y la Universidad del Rosario, ${ }^{6}$ en Venezuela en el año de 1975 bajo la dirección del Dr. Miguel Yáber y el Dr. Freddy Guevara, crean la primera unidad de perinatología en el Hospital Universitario de Caracas; en el año de 1984 se inicia como subespecialidad docente asistencial, actualmente ubicada en el piso $10 \mathrm{del}$ Hospital Universitario de Caracas, ${ }^{7}$ con el aval de la Universidad Central de Venezuela. ${ }^{8}$ 


\section{PERFIL ACADÉMICO DEL EGRESADO}

La residencia de perinatología tiene un pénsum académico el cual se desarrolla en dos años, con dedicación exclusiva, teniendo que cumplir con 41 materias, 138 créditos y tesis de grado. ${ }^{8}$

\section{1. Área asistencial}

Para que la asistencia perinatal sea integral y continua, es indispensable observar los aspectos preventivos, curativos y rehabilitadores de la salud; lo referente al bienestar psicológico, social y funcional de las mujeres en etapa reproductiva, y la población obstétrica en general. De tal manera que el profesional egresado es capaz de cumplir las siguientes actividades. ${ }^{8}$

\subsection{Preventivo}

Promoción y preservación de la salud. Para ello planifica, dirige y participa en actividades de promoción de la salud en los siguientes aspectos:

1.1.1 Educación sanitaria dirigida a la mujer embarazada utilizando métodos y estrategias de comunicación acordes con la población y con sus particulares necesidades.

1.1.2 Orientación a la comunidad a través de foros, talleres y conferencias, empleando, si fuere necesario, hasta los medios de comunicación.

1.1.3 La identificación y el asesoramiento sobre el riesgo preconcepcional de todas las mujeres en edad fértil y con deseos de embarazo.

1.1.4 Promoción de las actitudes de consideración y respeto hacia la embarazada por parte de la familia y de la comunidad.

\subsection{Curativo}

1.2.1 Asistencia médica y control a nivel de la consulta ambulatoria con el fin de realizar de forma temprana un diagnóstico de riesgo perinatal, y establecer una conducta y un manejo terapéutico precoz.

1.2.2 Control y seguimiento en la consulta de alto riesgo médico, obstétrico y perinatal, según la complejidad clínica del caso, donde se profundiza el diagnóstico mediante técnicas propias de cada nivel de atención y se ajustan conductas terapéuticas cónsonas con cada patología.

1.2.3 En casos de fetos portadores de malformaciones congénitas, está en la capacidad de realizar a la paciente y sus familiares, asesoramiento genético en busca de causas genéticas, hereditarias o ambientales, a los fines de establecer criterios de riesgos y recurrencias en embarazos subsiguientes.

1.2.4 Evaluación de control y seguimiento de las pacientes de alto riesgo hospitalizadas y su asistencia del preparto y parto.

1.2.5 Participación activa en las reuniones clínicas multidisciplinarias, en donde se discuta cada caso en particular.

1.2.6 Participación activa en las reuniones de epicrisis y del servicio de anatomía patología (AP) en su sección de AP perinatal (APP).

1.2.7 Asistir y participar en el seguimiento de los $\mathrm{RN}$ de alto riesgo en la unidad de terapia intensiva neonatal (UTIN).

1.2.8 Realizar y/o participar en la ejecución de procedimientos invasivos diagnósticos o de tratamientos.

\subsection{Rehabilitación y apoyo psicológico:}

1.3.1 De aquellas pacientes que por razones adversas y diversas, su embarazo no haya concluido en el final deseado.

1.3.2 Desarrollo de habilidades comunicacionales eficaces, con los fines de lograr un claro entendimiento con la paciente y sus familiares, acerca de la enfermedad que sufre la embarazada y/o su descendiente y de las repercusiones y/o limitaciones hacia el futuro.

1.3.3 Conocimiento amplio de los servicios disponibles para la debida orientación de la paciente de alto riesgo. 


\section{2. Áreas administrativas}

\section{Planificación y programación.}

2.1 Adquisición de las herramientas necesarias para la elaboración de planes de prevención y manejo de enfermedades tanto en el ámbito comunitario como institucional.

2.2 Desarrollo de las capacidades para conducir y administrar una unidad de perinatología, independiente del nivel de que se trate.

2.3 Conocimiento de las prácticas de atención primaria de salud.

2.4 Desarrollo de la capacidad de liderazgo.

\section{3. Área social y educativa}

3.1 Promoción de los mecanismos de prevención de las disfunciones sociales a nivel del individuo, de la familia y de la comunidad.

3.2 Promover la identificación y captación de líderes en la comunidad para lograr con ellos un efecto multiplicador.

3.3 Propiciar la integración, el manejo y la actualización de los miembros del equipo de salud.

3.4 Fomento de la participación de las agrupaciones públicas y privadas en la atención de las embarazadas de alto riesgo y del cuidado de sus hijos.

\section{4. Área de investigación.}

Para la realización de esta función deberá de aplicar el método científico con el objeto de estudiar y producir conocimiento en las áreas de su competencia y que se vinculen con su especialidad, para ello está en capacidad de:

4.1 Realizar investigaciones bibliográficas y socio epidemiológicas.

4.2 Participar, diseñar y ejecutar investigación en equipo.

4.3 Participar, diseñar y ejecutar en equipo, en el campo de la investigación experimental (Unidad de Cirugía Experimental).

4.4 Participar, diseñar y ejecutar en equipo planes de investigación de campo.
4.5 Conocer y respetar las normas que rigen la investigación científica tanto en seres humanos como en animales de experimentación.

4.6 Redactar informes y trabajos para exposiciones y publicaciones en congresos, cursos y seminarios.

4.7 Preparar y utilizar el material audiovisual.

4.8 Conocer y manejar adecuadamente las fuentes de información.

4.9 Leer y analizar críticamente un artículo en español o en inglés.

\section{NIVEL DE ENTRENAMIENTO:}

La Unidad de Perinatología, cuenta en estos momentos con una moderna planta física, tecnología de punta y un grupo docente asistencial altamente capacitado, dándole la oportunidad a la población de la realización de cualquier tipo de procedimiento diagnóstico y/o terapéutico, ubicándola como una de las más modernas a nivel mundial, además de ser creadora de nuevo conocimiento, mediante la investigación, para lo cual tiene diferentes convenios interinstitucionales y con el Instituto de Investigaciones Científicas de Venezuela en el campo de la terapia fetal in utero a cargo del doctor Rubén Quintero. ${ }^{8}$

Dentro del nivel organizativo de la unidad existe un manejo multidisciplinario, teniendo en una misma área servicios como hospitalización, consulta externa de alto riesgo médico y obstétrico, laboratorio clínico, sala de partos, asesoría preconcepcional, genética clínica y laboratorio de genética, unidad de terapia intensiva neonatal, unidad de terapia intensiva adultos, pediatría quirúrgica, anestesiología obstétrica, psiquiatría perinatal, cardiología infantil; además del apoyo de otras áreas del Hospital Universitario de Caracas, el cual es de IV nivel de complejidad.

Durante el año 2005, la unidad realizó 17.523 evaluaciones distribuidas así. (Cuadro 1).

Durante los dos años de residencia docente asistencial, cada residente tiene la posibilidad de realizar más de 3.100 evaluaciones (Cuadro 2) y de participar en el diagnóstico y manejo de aproximadamente 450 embarazos complicados con malformaciones fetales. 


\begin{tabular}{|l|c|}
\hline $\begin{array}{l}\text { Cuadro 1. Evaluaciones realizadas } \\
\text { en el año } 2005\end{array}$ \\
\hline \begin{tabular}{l} 
Screening ecográfico de primer trimestre \\
\hline Ecografía de detalle anatómico
\end{tabular} & 2.551 \\
\hline Ecografía pélvica transvesical & 8.756 \\
\hline Ecografía pélvica transvaginal & 90 \\
\hline Ecografía transfontanelar & 1.591 \\
\hline Ecografía renal & 521 \\
\hline Ecografía abdominal & 225 \\
\hline Pruebas de bienestar fetal (monitoreo y PBF) & 1.503 \\
\hline $\begin{array}{l}\text { Ecocardiografía fetal + Doppler } \\
\text { sión, cardiocentesis, etc.) }\end{array}$ & 637 \\
\hline Evaluaciones de alto riesgo obstétrico & 845 \\
\hline Pruebas de B - hCG & 209 \\
\hline Procedimientos invasivos (Amniocentesis, & 450 \\
\hline
\end{tabular}

* Cirugía fetal intrauterina comprendida entre enero del 2003 y junio del 2006.

Cuadro 2. Actividades por residente durante los dos años de la subespecialidad

\section{Actividades durante 2 años de residencia}

\begin{tabular}{|l|c|}
\hline Screening ecográfico de primer trimestre & 305 \\
\hline Ecografía de detalle anatómico & 1.515 \\
\hline Ecografía pélvica transvesical & 19 \\
\hline Ecografía pélvica transvaginal & 352 \\
\hline Ecografía transfontanelar & 50 \\
\hline Pruebas de bienestar fetal & 478 \\
\hline Ecocardiografía fetal + Doppler & 194 \\
\hline Evaluaciones de alto riesgo obstétrico & 96 \\
\hline Procedimientos invasivos & 110 \\
\hline
\end{tabular}

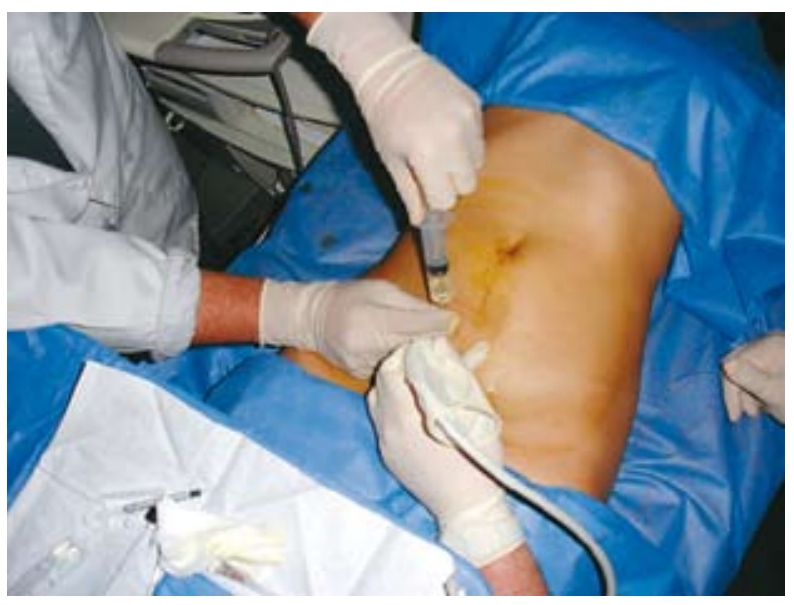

Figura 1. Realización vesicocentesis fetal para pruebas de función renal, feto de 17 semanas con diagnóstico de valva uretral posterior.

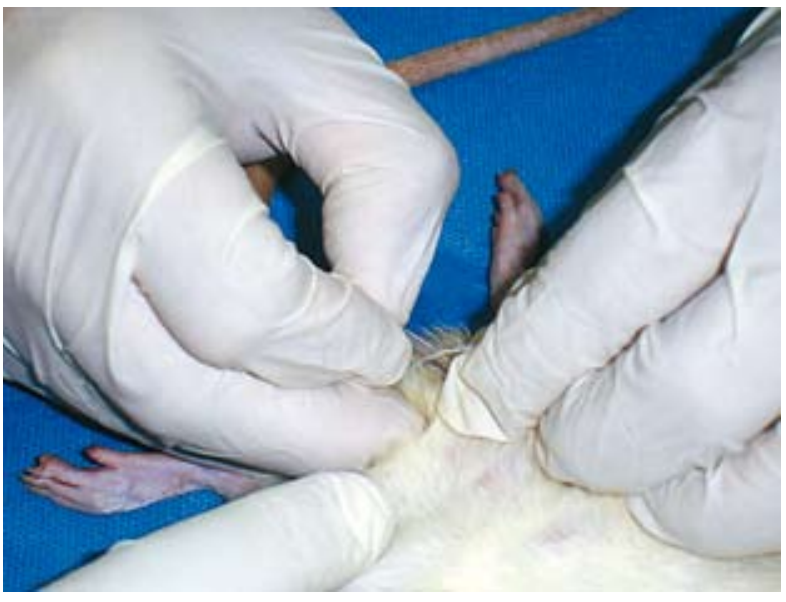

Figura 2. Investigación en animales: cateterización de uretra en rata sprague dawley, Instituto de Cirugía Experimental - Instituto Venezolano de Investigaciones Científicas.

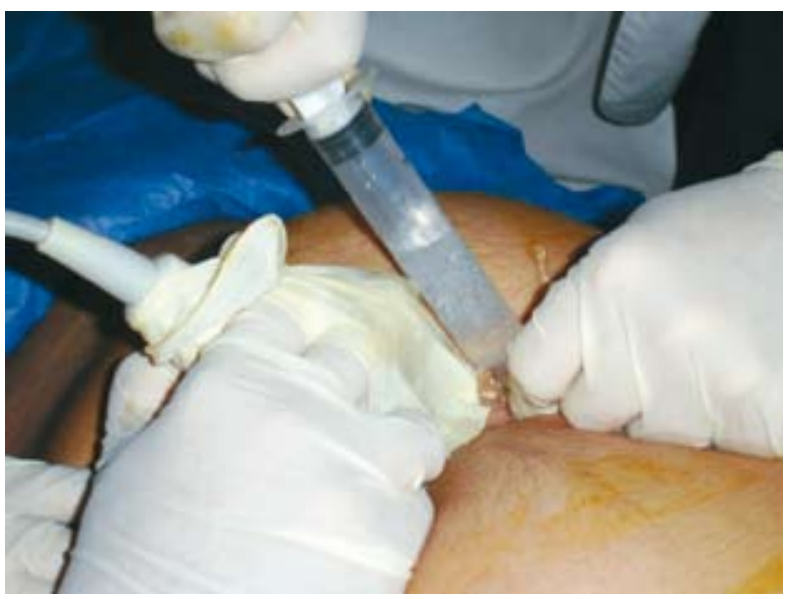

Figura 3. Lesión quística ovario fetal derecho de $73 \mathrm{~mm} *$ $52 \mathrm{~mm}$ : punción quiste ovario fetal, feto 32 semanas. 

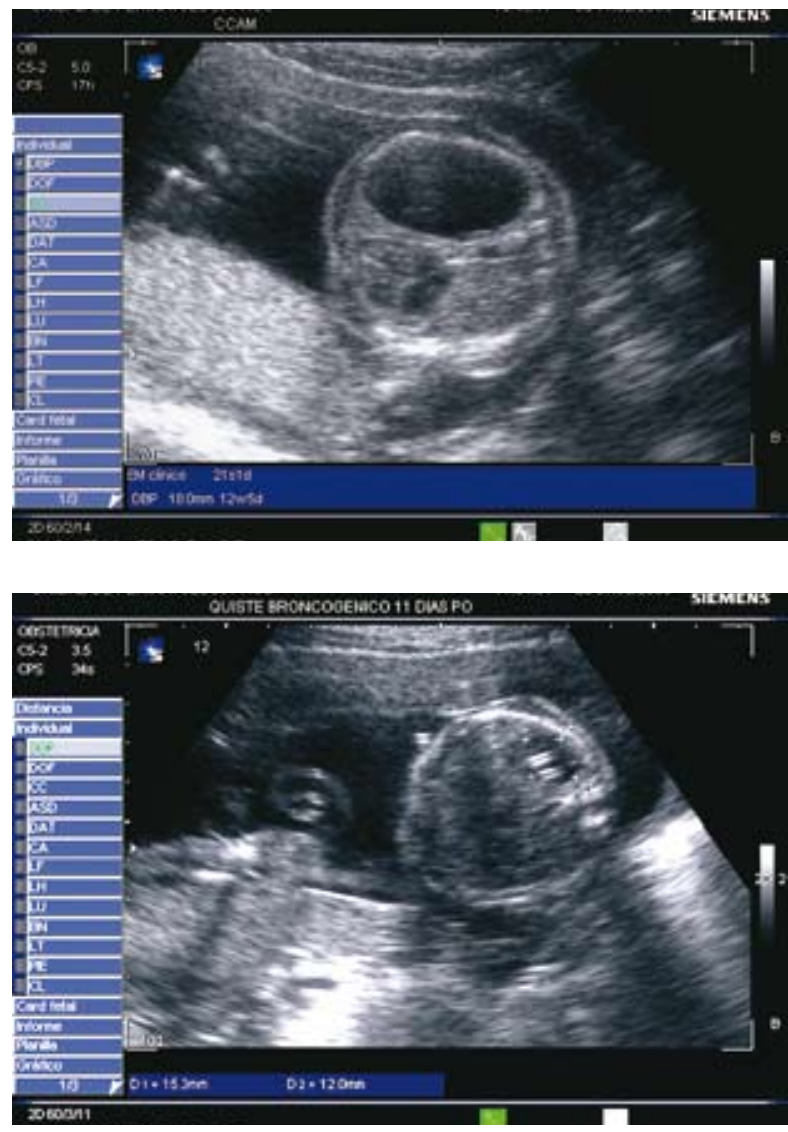

Figura 4. Pre y posoperatorio, colocación catéter de Harrison, feto de 22 semanas con diagnóstico enfermedad adenomatoidea quística pulmonar Tipo I.

\section{COMENTARIO}

Aunque no existe la cantidad de procedimientos que debe realizar un residente para obtener un adecuado entrenamiento en medicina materno fetal, sí existen actividades mínimas para optar al título de perinatólogo o especialista en medicina materno fetal dadas por la American Board of Obstetrics and Gynecology (ABOG). ${ }^{9}$

Según la ABOG, debe estar en capacidad de realizar cesáreas, histerectomía de emergencia, procedimientos de esterilización, cerclaje de cérvix, partos vaginales instrumentados, manejo de embarazos de alto riesgo, técnicas para control de hemorragia, intubación neonatal, pruebas de bienestar fetal anteparto e intraparto, amniocentesis, cateterización de la arteria pulmonar, evaluación materna y fetal por ultrasonografía, interrupción de embarazos (excepto donde no es permitido por la ley), procedimientos invasivos diagnósticos y terapéuticos, asesoría genética, biopsia de vellosidades coriónicas, versión cefálica externa, versión externa o interna del segundo gemelo, manejo de distocias, parto vaginal de embarazos múltiples. ${ }^{9-12}$

En el Hospital Universitario de Caracas por ser centro de referencia de toda Venezuela y algunos países vecinos, se tiene la oportunidad de manejar un número alto de pacientes, se cuenta con una de las unidades más importantes de cirugía fetal del mundo y actualmente se están formando subespecialistas de otros países para poder integrar a Latinoamérica.

Bajo el concepto del feto como paciente, el perinatólogo de la unidad está en capacidad de prestar servicios de salud en aspectos preventivos, médicos, quirúrgicos y de rehabilitación en sus áreas de especialización: asesoría preconcepcional, diagnóstico prenatal, diagnóstico citogenético prenatal, tamización e cromosomopatías, pruebas serológicas, asesoría genética, control de embarazos de alto riesgo obstétrico y médico, pruebas de bienestar fetal, monitorización de los casos de riesgo en área de emergencia obstétrica, diagnóstico oportuno de noxas que pueden en su evolución ameritar cirugía fetal, participar en trabajos de investigación en humanos, animales y docencia universitaria.

Desafortunadamente existen pocos centros para la formación de perinatólogos (2 años) en Latinoamérica, por lo cual, los cursos de profundización pueden ser una opción válida hasta no tener suficientes cupos por año para la formación de subespecialistas en medicina materno fetal y de esta forma disminuir los índices de morbilidad y mortalidad materno fetal.

\section{REFERENCIAS}

1. Secretaría de Salud. Manual de organización específico. México: Instituto Nacional de Perinatología; 2005.

2. Universidad Nacional de Colombia, Consejo académico. Acuerdo No 044 del 2004, especialidad en perinatología y neonatología de la facultad de medicina de la Universidad Nacional. Bogotá, Colombia; 2004. 
3. Sociedad Iberoamericana de Diagnóstico y Tratamiento Perinatal, SIADTP. Estatutos de la SIADTP. Barcelona, España; 2004.

4. Federación Latinoamericana de Sociedades de Obstetricia y Ginecología, FLASOG. Disponible en: http://www.flasog.org.

5. The Fetal Medicine Foundation. Diploma in Fetal Medicine. London, U.K. Disponible en: http://www. fetalmedicine.com.

6. Johns Hopkins Medicine. The Department of Gynecology and Obstetrics Perinatology (Maternal Fetal Medicine). Disponible en: http://www.hopkinsmedicine.org.

7. Organización Panamericana de la Salud, Oficina Regional de la Organización Mundial de la Salud, Centro Latinoamericano de Perinatología Salud de la Mujer y Reproductiva, CLAP. Montevideo, Uruguay: Universidad de la República. Estatutos CLAP; 2001.
8. International Federation of Gynecology and Obstetrics, FIGO. Disponible en: http://www.figo.org.

9. Universidad Central de Venezuela, Facultad de Medicina, Hospital Universitario de Caracas, Cátedra de obstetricia y ginecología. Programa del Curso de Especialización en Medicina Perinatal. Caracas, Venezuela: Hospital Universitario de Caracas; 2006.

10. Unisánitas. Especialización en Medicina Materno Fetal, Programa de Especialización en Medicina Materno Fetal (Clínica Colsánitas - Universidad del Rosario). Bogotá, Colombia. Disponible en: http://www.unisanitas.edu. $\mathrm{co} / \mathrm{ummf} / \mathrm{html}$

11. The American Board of Obstetrics and Gynecology, Inc. General and special requirements for graduate medical education in the subspeciality area of maternal fetal medicine. Dallas, TX: The Vineyard Centre; 2005.

12. Estatuto de la Sociedad de Obstetricia y Ginecología de Venezuela. Décima Revisión. Caracas; 2002.

\section{Conflicto de intereses: ninguno declarado.}

